\title{
Renal cell carcinoma presenting as a simple renal cyst: A case report
}

\author{
YANLAN YU, LIANG MA, ZHENGHUI WANG and ZHIGEN ZHANG \\ Department of Urology, Sir Run-Run Shaw Hospital, College of Medicine, \\ Zhejiang University, Hangzhou, Zhejiang 310016, P.R. China
}

Received August 22, 2016; Accepted January 19, 2017

DOI: $10.3892 / \mathrm{mco} .2017 .1173$

\begin{abstract}
Cases of renal cell carcinoma (RCC) presenting as a simple cyst are extremely rare. We herein report the case of a patient with RCC diagnosed as a simple renal cyst preoperatively. A 39-year-old female patient presented with abdominal pain for 3 months. Ultrasonography and contrast-enhanced computed tomography revealed a simple cyst in the left kidney. The patient underwent laparoscopic decortication of the renal cyst. Biochemical analysis of the cystic fluid revealed unusually low levels of potassium, sodium, calcium and glucose, and the histological examination of the floor of the cyst indicated malignancy. Laparoscopic nephrectomy was performed 20 days later and the pathological examination confirmed the diagnosis of RCC of the clear cell type. At the 2-year follow-up, the patient remained well and recurrence-free on imaging. The aim of the present study was to emphasize the importance of recognizing that RCC may occur in what appears to be a simple renal cyst based on imaging results. Biochemical analysis of the cystic fluid may help identify the presence of malignancy.
\end{abstract}

\section{Introduction}

Simple renal cysts are the most common renal masses in the urinary tract. The majority of these cysts are benign, asymptomatic, and are usually treated conservatively. The major clinical concern is distinguishing simple renal cysts from complex cysts that may harbor malignancy. Recent advances in radiology have improved the clinical approach to asymptomatic renal masses, including benign cysts and renal cell carcinoma (RCC), by ultrasound (US), computed tomography (CT) or magnetic resonance imaging. Clinically, the classification of renal cystic tumors is based on the Bosniak classification system $(1,2)$. In Bosniak category I, the incidence

Correspondence to: Dr Liang Ma, Department of Urology, Sir Run-Run Shaw Hospital, College of Medicine, Zhejiang University, 3 East Qingchun Road, Hangzhou, Zhejiang 310016, P.R. China E-mail: srrshurology@163.com

Key words: renal cell carcinoma, simple renal cyst, cyst fluid of malignancy is very low. In categories II and III, a certain extent of malignant cystic disease is included and adequate treatment may be required. Renal cysts with thickened, irregular or smooth walls or septa and enhancement following contrast injection, favor malignancy.

Case reports of RCCs presenting as simple cysts are extremely rare. It is difficult to determine preoperatively whether or not a cyst is malignant. Although cytology and serum tumor markers have also been investigated, their diagnostic value is limited to cases of renal malignancy (3). We herein describe the case of a patient with transformation of a simple renal cyst to RCC.

\section{Case report}

A 39-year-old female patient with left flank pain for 3 months was referred to the Sir Run-Run Shaw Hospital (Hangzhou, China). The patient had no underlying predisposing conditions or syndromes, such as a history of trauma, chronic renal disease, or tuberous sclerosis. There was also no family history of renal cysts, RCC, or renal malformations. The results of the laboratory investigations were normal. US examination revealed a 70x60-mm cyst in the upper pole of the left kidney and an abdominal contrast-enhanced CT scan showed a simple cyst in the lateral aspect of the left kidney (Fig. 1). The density of the cyst contents was similar to that of water and there was no enhancement. The patient underwent laparoscopic decortication of the cyst. Under laparoscopic visualization, the cystic fluid was aspirated by a syringe and immediately sent for biochemical analysis. The cystic fluid was clear and yellow, and the biochemical analysis showed that the aspirated fluid contained extremely low levels of potassium, sodium, calcium and glucose (Table I). This was quite different from the contents of regular cysts, which usually have a consistency similar to that of serum. The cyst wall was excised and sent for pathological evaluation and the histological examination of the floor of the cyst indicated malignancy. Macroscopically, the external surface of the cyst was smooth, but small excrescences were observed in the inner wall of the cyst. On immunostaining, the tumor cells stained positive for RCC marker, CD10 and vimentin, and negative for cytokeratin 7 (Fig. 2). Laparoscopic nephrectomy was performed 20 days after decortication and the pathological examination confirmed the diagnosis of RCC of the clear cell type. The postoperative course was uneventful. 
Table I. Biochemical analysis of the cystic fluid.

\begin{tabular}{lcccccc}
\hline $\begin{array}{l}\text { Potassium } \\
(\mathrm{mmol} / \mathrm{l})\end{array}$ & $\begin{array}{c}\text { Sodium } \\
(\mathrm{mmol} / \mathrm{l})\end{array}$ & $\begin{array}{c}\text { Chlorine } \\
(\mathrm{mmol} / \mathrm{l})\end{array}$ & $\begin{array}{c}\text { Calcium } \\
(\mathrm{mmol} / \mathrm{l})\end{array}$ & $\begin{array}{c}\text { Blood urea nitrogen } \\
(\mathrm{mmol} / \mathrm{l})\end{array}$ & $\begin{array}{c}\text { Creatinine } \\
(\mu \mathrm{mol} / \mathrm{l})\end{array}$ & $\begin{array}{c}\text { Glucose } \\
(\mathrm{mmol} / \mathrm{l})\end{array}$ \\
\hline$<1.00$ & $<100$ & $<50$ & 0.87 & 2.40 & 22 & 1.94 \\
\hline
\end{tabular}

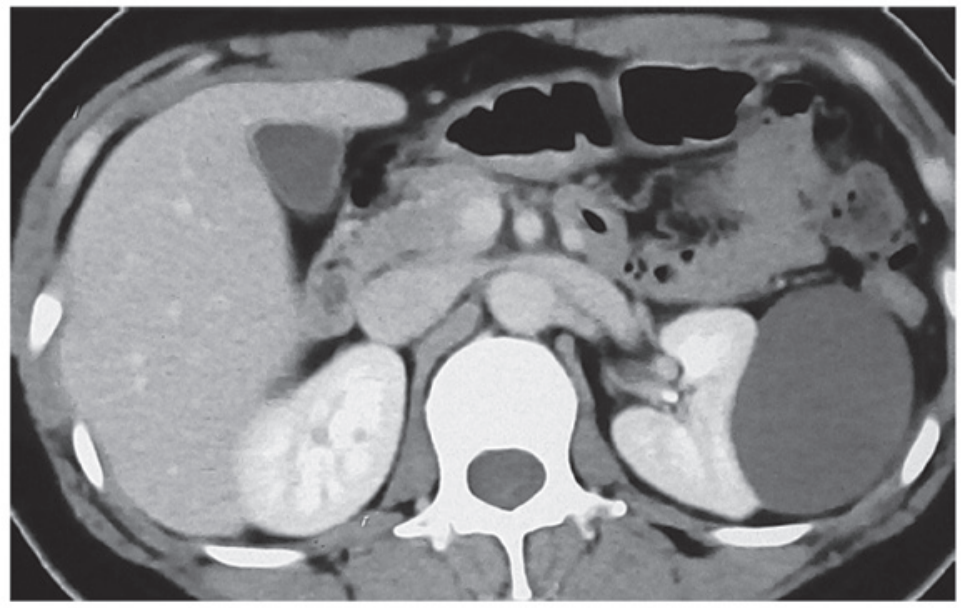

Figure 1. Abdominal computed tomography scan with contrast showing a simple cyst sized 7x6x6 cm in the left kidney, with a density of 2 Hounsfied units.
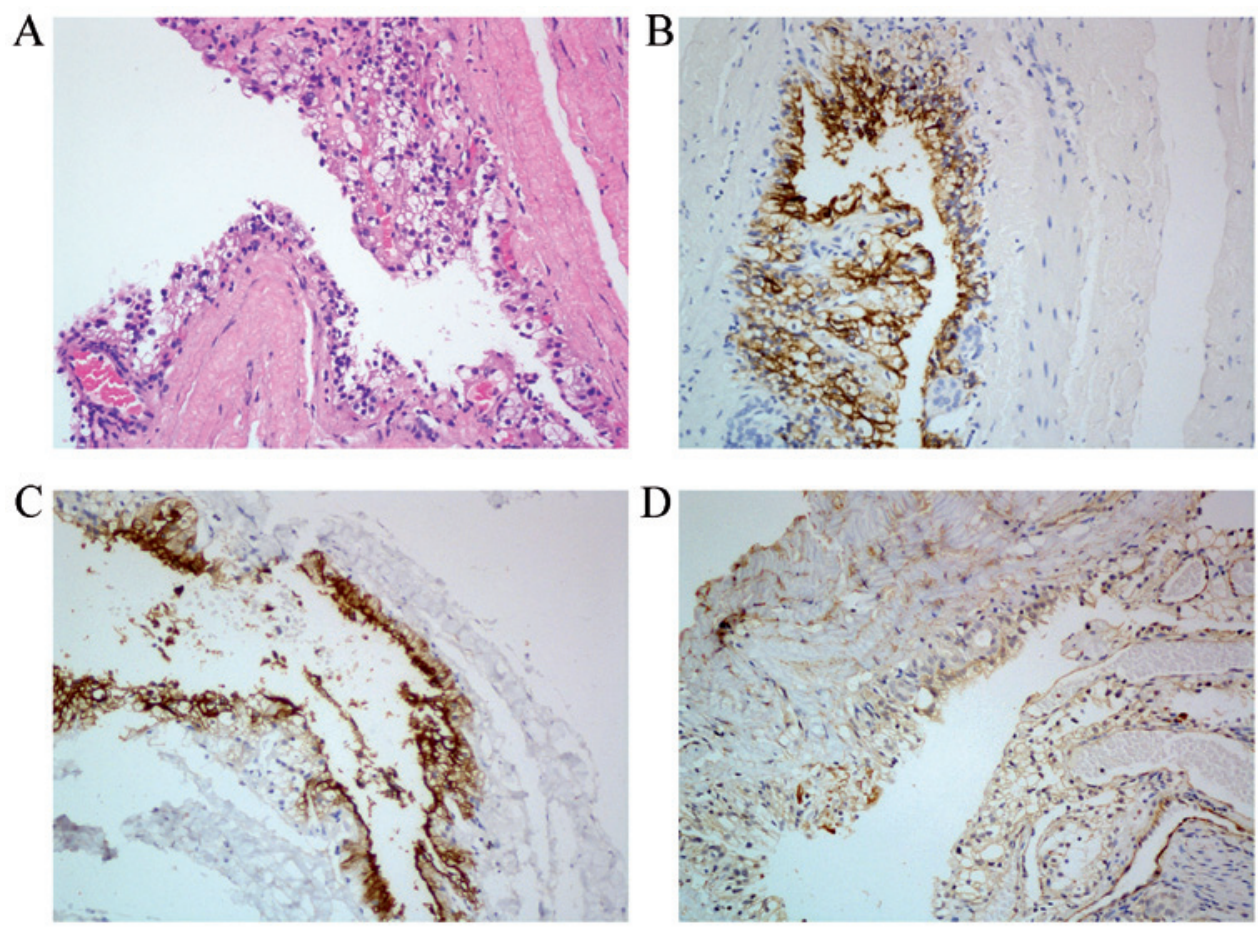

Figure 2. Histological findings of the tumor. (A) Hematoxylin and eosin staining of the tumor showing clear cells with relative small nuclei forming alveolar structures; (B) imunohistochemical staining for renal cell carcinoma marker; (C) imunohistochemical staining for CD10; (D) Imunohistochemical staining for vimentin (all magnifications, $\mathrm{x} 200$ ).

The patient was followed up by chest X-ray, liver function tests, abdominal CT scans and physical examination every 3 months for the first year and every 6 months thereafter. There has been no evidence of local recurrence or metastatic disease to date.

\section{Discussion}

RCC presenting as a simple renal cyst is extremely rare and only few cases have been reported to date in the English literature. Lin et al (4) reported a patient with flank pain who 
was initially diagnosed with a simple cyst and was treated by ultrasound-guided aspiration, but the pain developed again 3 weeks later and ultrasound examination indicated a recurrent sizeable cyst. Nephrectomy was then performed and pathological examination confirmed the diagnosis of papillary RCC. Visapää et al (5) evaluated 482 patients who underwent partial or radical nephrectomy for kidney tumors and demonstrated an association between simple renal cysts and papillary RCC. Sakai et al (6) reported the case of a simple renal cyst that developed into a septated renal cyst and finally, to cystic RCC of the clear cell type, over a period of 6 years. That case demonstrated the natural history of malignant transformation from a simple renal cyst, and emphasized that careful follow-up of renal cysts, particularly of complicated cysts, is mandatory for successful treatment of RCC. Furthermore, in the Japanese literature, Takao et al (7) also reported a cystic RCC diagnosed as a simple cyst preoperatively.

Diagnosing a simple renal cyst is generally not difficult. The Bosniak classification system has been accepted as a method for diagnosing and determining the management of cystic renal masses, which was designed to analyze the morphology of cystic masses based solely on CT findings. However, it is difficult to determine preoperatively whether or not a cyst is malignant. Our patient, who initially presented with a simple cyst on CT imaging, apparently classified as Bosniak category I, was pathologically diagnosed with RCC. This may be due to the small size of the malignant tissue inside the cyst, which cannot be detected by imaging examinations alone.

The management options for renal cysts include radiological surveillance, ultrasound- or CT-guided cyst aspiration or biopsy and laparoscopic exploration or open surgical exploration, with or without radical or partial nephrectomy. Cyst aspiration for cytology may be helpful in the management of a patient only if malignant cells are clearly seen. Radical or partial nephrectomy may result in overtreatment, as nephrectomies often prove unnecessary. Omachi et al (8) reviewed the characteristics of intracystic fluid associated with RCC from the Japanese literature and found that cytological examination was negative for tumor cells in the majority of these cases, and $70 \%$ was hemorrhagic intracystic fluid. CT-guided biopsy may also be difficult and occasionally leads to misdiagnosis $(9,10)$.

In the present case, the renal lesion was found to be a simple cyst on ultrasound and CT examination, classified as Bosniak category I. The possibility of malignancy appeared to be extremely low, but finally pathology demonstrated RCC. Interestingly, the biochemical analysis of the aspiration fluid revealed that it contained low levels of potassium, sodium, calcium and glucose, which is unusual in a simple renal cyst. In the case reported by Lin et al (4) the cystic RCC also had low glucose $(0.333 \mathrm{mmol} / \mathrm{l})$, and increased lactate dehydrogenase $(2,361 \mathrm{U} / 1)$ levels. In our institute, the cystic fluid is routinely tested in all patients who undergo laparoscopic decortication, in order to detect whether the cyst communicates with the renal pelvis, and we observed that the consistency of the contents of simple renal cysts is almost the same as that of the serum (data not shown). The change of biochemical consistency and protein levels may be associated with the microenvironment changes in malignancy.

Therefore, it is crucial that we recognize the possibility of RCC appearing as a simple renal cyst. Biochemical analysis of the cystic fluid may help identify the presence malignancy and we recommend that biochemical analysis of the cystic fluid be routinely performed, either by ultrasound- or CT-guided cyst aspiration or during laparoscopic procedures. If suspicious, achieving a rapid pathological diagnosis by biopsy of the cyst wall is necessary. If the presence of malignancy is pathologically confirmed, an immediate partial or radical nephrectomy may be performed under the same anesthetic as the laparoscopic procedure.

\section{References}

1. Bosniak MA: The current radiological approach to renal cysts. Radiology 158: 1-10, 1986.

2. Israel GM and Bosniak MA: An update of the Bosniak renal cyst classification system. Urology 66: 484-488, 2005.

3. Li G, Bilal I, Gentil-Perret A, Feng G, Zhao A, Peoc'h M, Genin C, Tostain J and Gigante M: CA9 as a molecular marker for differential diagnosis of cystic renal tumors. Urol Oncol 30: 463-468, 2012

4. Lin CJ, Chen YC, Chen HH, Wu CJ and Hsu JM: Renal cell carcinoma presenting as a huge simple renal cyst. Med Oncol 25: 104-106, 2008.

5. Visapää H, Glucker E, Haukka J, Taari K and Nisen H: Papillary renal cell cancer is strongly associated with simple renal cysts. Urol Int 91: 269-272, 2013.

6. Sakai N, Kanda F, Kondo K, Fukuoka H and Tanaka T: Sonographically detected malignant transformation of a simple renal cyst. Int J Urol 8: 23-25, 2001.

7. Takao T, Gotoh T, Takada S and Sugao H: Cystic renal cell carcinoma diagnosed as a simple cyst preoperatively with incidental renal tumor: A case report. Hinyokika Kiyo 45: 339-342, 1999 (In Japanese).

8. Omachi T, Sakamoto W, Kishimoto T, Kawano M, Oyama A, Kamizuru M, Maekawa M, Hagihara S and Nakamura K: A case of renal cyst associated with renal cell carcinoma-characteristics of intracystic fluid associated with renal cell carcinoma from Japanese reports. Hinyokika Kiyo 38: 323-326, 1992 (In Japanese).

9. Siegel CL: Accuracy of diagnosis by guided biopsy of renal mass lesions classified indeterminate by imaging studies. J Urol 165: 322-323, 2001.

10. Kadekawa K, Miyazato M, Saito S, Morozumi M, Matsuzaki A, Yoshimi N and Sugaya K: Renal cell carcinoma originating in a renal cyst in a 12-year-old girl. J Pediatr Surg 44: e5-e7, 2009. 